\section{Pilates as physiotherapy in patients with Parkinson disease: a pilot study}

\author{
José Maria Cancela ${ }^{1,2}, \mathrm{PhD}$; Gustavo Rodriguez ${ }^{3}, \mathrm{PhD}$; Iris Machado ${ }^{3}, \mathrm{PhD}$ \\ Irimia Mollinedo ${ }^{4}$, MSc
}

\begin{abstract}
Background. Pilates improves core muscle function and lumbopelvic stability. The basic principles of Pilates are concentration, control, centring, diaphragmatic breathing, lightness, precision, strength, and relaxation. This study aims to determine the effects of Pilates on clinical symptoms and static balance in patients with Parkinson disease (PD).

Methods: Of 36 patients with PD, 15 (mean age, 73.5 years) who had a Hoehn and Yahr stage of 1 to 3 and a stable reaction to anti-Parkinson medication were randomly allocated to the Pilates group $(n=8)$ or conventional exercise group $(n=7)$. Participants were assessed 1 week before intervention (week 0 ) and 1 and 4 weeks after intervention completed (weeks 15 and 18). Motor and non-motor impairment and disability were assessed using the Spanish version of the Movement Disorder Society Unified Parkinson's Disease Rating Scale (MDSUPDRS). Static balance was assessed using the Stabilometric platform. Participants were instructed to stand centrally on the platform with feet hip-width apart and arms at their sides and with open eyes and closed eyes each for $30 \mathrm{~s}$. Parameters recorded included centre of pressure of the body in the frontal plane (COP X), centre of pressure of the body in the sagittal plane (COPY), speed of oscillation of the centre of pressure in the sagittal plane (forward-backward) [F-B speed], speed of oscillation of the centre of pressure in the frontal plane (mediumlateral) [M-L speed], and displacement area of the centre of pressure (ellipse area).
\end{abstract}

Results: All 15 participants attended over $80 \%$ of the sessions. From pre-intervention to post-intervention, participants in the Pilates group had significant improvements in MDS-UPDRS, open eyes COP $\mathrm{X}$, open eyes COPY, open eyes ellipse area, and closed eyes COPY. Whereas participants in the conventional exercise group had significant improvement in open eyes COP Y and closed eyes COP Y but had significant deterioration in open eyes F-B speed and closed eyes F-B speed. Compared with the conventional exercise group, the Pilates group had significantly greater improvement in all parameters except for body mass index, closed eyes ellipse area, and M-L speed. From post-intervention to follow-up, participants in both groups lost most of the improvement in MDS-UPDRS and in static balance parameters. Compared with the conventional exercise group, the Pilates group had significantly greater improvement in MDS-UPDRS, open eyes COP X, closed eyes COP X, and closed eyes COPY.

Conclusion: Pilates exercise is a useful rehabilitation strategy for people with mild to moderate PD, with a positive effect on motor impairment and static balance.

Key words: Exercise; Neurodegenerative diseases; Parkinson disease; Postural balance.

\section{ORIGINAL ARTICLE}

\author{
Department of Special Didactics, \\ Faculty of Education and Sport Science, \\ University of Vigo, Pontevedra, Spain \\ 2 Galicia Sur Health Research Institute (IIS \\ Galicia Sur), Sergas-UVIGO, HealthyFit \\ Research Group, Spain \\ 3 Department of Functional Biology \\ and Health Sciences, Faculty of \\ Physiotherapy, University of Vigo, \\ Pontevedra, Spain \\ 4 Galician Government, Galicia, Spain
}

Correspondence to: José María Cancela, Faculty of Education and Sport Science, University of Vigo- Campus da Xunqueira s/n 36005, Pontevedra, Spain. Email: chemacc@uvigo.es 


\section{INTRODUCTION}

Parkinson disease (PD) is a progressive neurodegenerative disorder characterised by motor, cognitive, affective, autonomic, and sensory impairments secondary to selective degeneration of neurons at multiple levels of the central and peripheral nervous systems. ${ }^{1}$ The cardinal features of PD are bradykinesia, rigidity, tremors, and gait impairment; all these lead to progressive loss of functional independence. ${ }^{2}$ Altered balance and postural control are disabling and poorly responsive to standard pharmacological treatment for motor symptoms. ${ }^{3}$

Exercise is a useful non-pharmacological alternative to improve postural instability, motor impairment, and balance task performance, leading to a slower progression of PD. ${ }^{4-6}$ Various types of exercise in or out of water such as aerobic exercise, gait training, balance training, and progressive resistance training have shown beneficial effects on different symptoms (motor and nonmotor)..$^{5-7}$ Aquatic activity is safe and beneficial for motor function, mobility, balance, flexibility, and cardiorespiratory endurance in patients with $\mathrm{PD}$, but more randomised controlled studies are needed to ratify this..$^{8,9}$ Further research that includes a follow-up phase and objective balance measurements is needed. ${ }^{10}$

Pilates can improve balance of persons with neurological conditions. ${ }^{11}$ Pilates improves flexibility and axial stability through strengthening the core musculature. Pilates is based on coordinated movement sequences rather than simple repetitive movements as in other exercises. ${ }^{12}$ Pilates is a useful rehabilitation strategy for PD. ${ }^{13}$ Short-term Pilates exercises can significantly improve walking and balance in older adults. ${ }^{14}$ Both Pilates and balance exercises have significant effects on balance, especially dynamic balance, on unstable surfaces among old women, but Pilates is safer and simpler to learn. ${ }^{15}$ In older patients with PD who have higher rates of imbalance and falls, such exercises can improve motor functions and balance. ${ }^{16}$

To the best of our knowledge, only one study reported on the effects of Pilates on balance in patients with $\mathrm{PD}$, but the study had a small sample size and no conventional exercise group. ${ }^{17}$ Other studies combined Pilates as part of exercise interventions, and specific effects were not provided. $^{18,19}$ Two studies that applied Pilates as strength training reported significant effects on lower limb strength, dynamic balance, and quality of life in patients with PD. ${ }^{20,21}$ We believe that Pilates has a significant effect on static balance, because it strengthens core muscles and increases awareness of them. This study aims to determine the effects of Pilates on clinical symptoms and static balance in patients with PD.

\section{METHODS}

This study was approved by the Ethics Committee of the University of Vigo, Pontevedra, Spain (reference: EC2015/484). Informed consent was obtained from each participant. Of 36 patients with PD recruited from the Provincial Parkinson Association of Pontevedra, Spain, 15 who had a Hoehn and Yahr stage of 1 to 3 (mean, 2.40; standard deviation, 0.65) and a stable reaction to anti-Parkinson medication were included. They were randomly allocated to the Pilates group $(n=8)$ or conventional exercise group $(n=7)$ [Figure 1]. The sample size was calculated based on a study on static balance $(\mathrm{d}=1.86, \mathrm{r}=0.68) .{ }^{11}$ To achieve a statistical power of 0.80 and a significance level of 0.05 , six patients with PD in each group were required. Assuming a 20\% dropout rate, at least 14 participants were required.

Participants were assessed 1 week before intervention (week 0 ) and 1 and 4 weeks after intervention completed (weeks 15 and 18). Neither group took physical exercise during the follow-up period.

Participants were assessed for motor and nonmotor impairment and disability 2 hours after taking PD medications by a physiotherapist using the Spanish version of the Movement Disorder Society Unified Parkinson's Disease Rating Scale (MDSUPDRS). ${ }^{22}$ The MDS-UPDRS comprises four parts: non-motor aspects of experiences of daily living (13 items), motor aspects of experiences of daily living (13 items), motor examination (18 items), and motor complications (6 items). Each item scores from 0 (normal) to 4 (severe). The total score ranges from 0 to 200; higher scores indicate greater PD symptoms.

Participants were assessed for static balance during the 'on' phase (1 to 1.5 hours after taking PD 


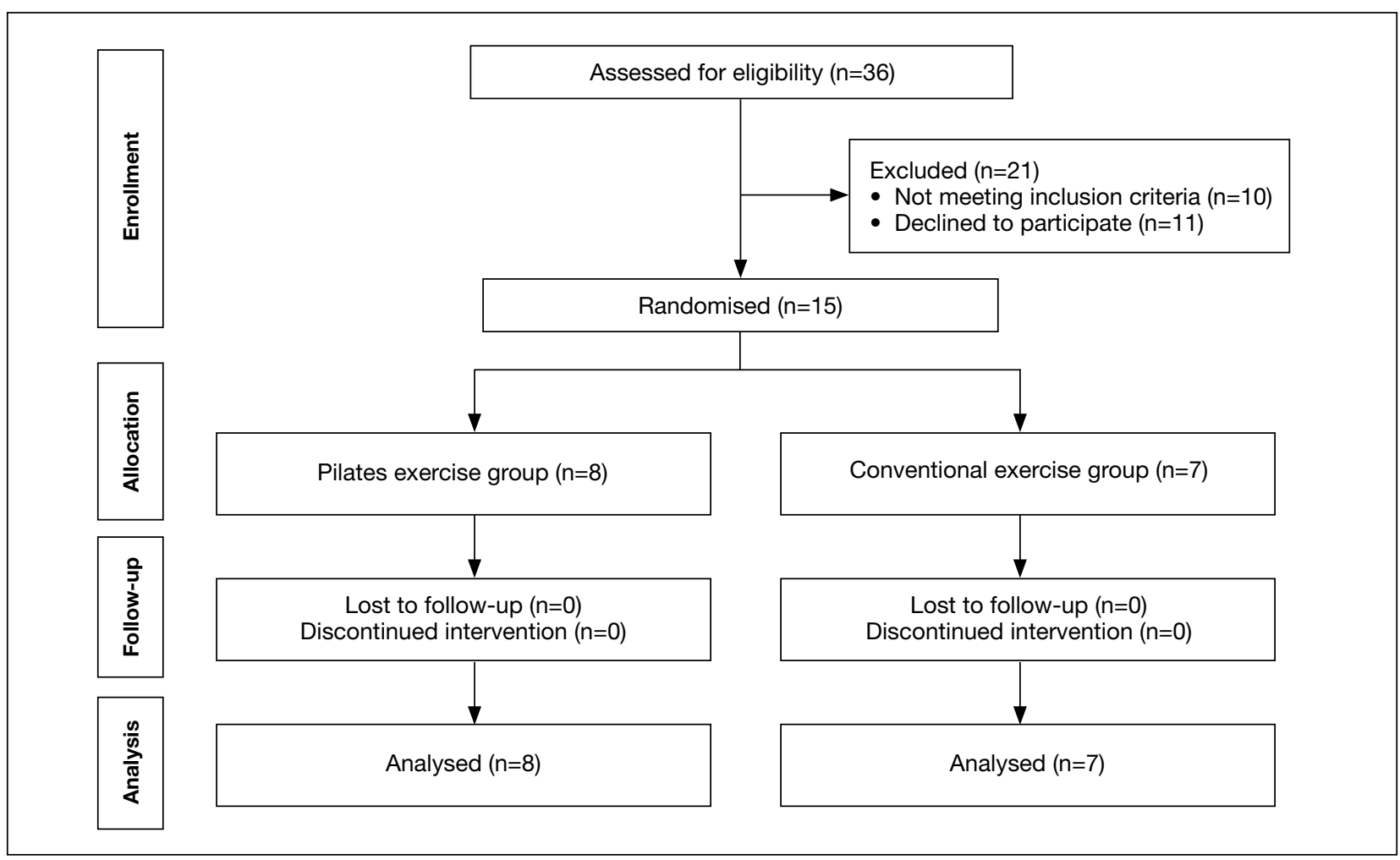

FIGURE 1. Flowchart of recruitment of participants

medications) by two physiotherapists specialised in neurological rehabilitation using the Stabilometric platform (TecnoBody PROKIN PK 3 P, Bergamo, Italy). The platform has a diameter of $40 \mathrm{~cm}$, with three strain gauges placed at $120^{\circ}$. These sensors are auto-calibrated and have $50 \mathrm{~g}$ of maximal resolution. Data were acquired in a frequency of $20 \mathrm{~Hz}$ and presented as a statokinesiogram, an axial stabilogram, and a Fourier transformation. Participants were instructed to stand centrally on the platform with feet hip-width apart and arms at their sides and with open eyes and closed eyes each for $30 \mathrm{~s}$. The mean of three attempts was recorded, with a 60-s interval of rest in between. ${ }^{17}$ Parameters recorded included (1) centre of pressure of the body in the frontal plane (COP X), (2) centre of pressure of the body in the sagittal plane (COP Y), (3) speed of oscillation of the centre of pressure in the sagittal plane (forwardbackward) [F-B speed], (4) speed of oscillation of the centre of pressure in the frontal plane (mediumlateral) [M-L speed], and (5) displacement area of the centre of pressure (ellipse area). Measurements further away from the centre indicate worse static stability. ${ }^{17}$
In the first 2 weeks of intervention, participants received two sessions each week of conventional exercise for 50 minutes to improve their aerobic capacity, muscular resistance, balance, and flexibility. The exercise combined land-based and water-based sessions and has been applied by the association since 2016. ${ }^{23}$ During the second week, participants were able to go from standing to sitting and from sitting to lying with help. In the third week, participants were randomised to receive Pilates exercise or conventional exercise for the following 12 weeks.

The Pilates exercise was developed and supervised by two experienced physiotherapists. Two sessions (one on a mat and the other in standing and sitting positions) each of 60 minutes were performed per week. ${ }^{20}$ Because of mobility deficits of patients, the Pilates exercise was designed to not generate continuous changes in body position. Participants started with warm-up exercises (for 5 minutes) to enable stimulation of bodily awareness, lateral ribcage breathing, correct alignment of the cervical spine, and neutral pelvic placement, followed by strengthening exercises for transversus 
abdominis and pelvic floor muscles. The main part of the session included 45 minutes of strength, mobility, and coordination exercises for upper and lower limbs in different positions. Intensity was progressively increased by adding/removing elastic bands to increase resistance or remove assistance to the movement. Each exercise included one set of three to five repetitions. No rest time was allowed between repetitions, as two exercises of the same muscle group were never performed in a row, and exercises alternated between the upper limbs and the lower limbs. At the end of the session, 10 minutes of cooling down activities such as gentle stretching and deep breathing exercises were carried out.

The Shapiro-Wilk test was used to verify normal distribution. The unpaired Student's $t$-test was applied to check the homogeneity of the sample; the two groups differed in baseline open eyes COP Y, closed eyes COP Y, and open eyes ellipse area. The paired Student's t-test was applied to evaluate intragroup differences in changes from pre- to postintervention and from post-intervention to followup. The analysis of variance was used to determine the intergroup differential effect. A $p$ value of $<0.05$ was considered statistically significant.

\section{RESULTS}

All 15 participants attended over $80 \%$ of sessions. The Pilates and conventional exercise groups were comparable in terms of sociodemographic characteristics (TABLE $\mathbf{I}$ ), but the two groups differed in baseline open eyes COPY, closed eyes COPY, and open eyes ellipse area.

From pre-intervention to post-intervention, participants in the Pilates group had significant improvements in MDS-UPDRS (58.38 \pm 18.01 vs $43.25 \pm 11.07, p<0.001)$, open eyes COP $X$ $(-3.28 \pm 13.28 \mathrm{~mm}$ vs $-0.75 \pm 10.44 \mathrm{~mm}, \mathrm{p}=0.032)$, open eyes COP Y $(6.19 \pm 21.80 \mathrm{~mm}$ vs $-3.13 \pm 21.73 \mathrm{~mm}$, $\mathrm{p}<0.001)$, open eyes ellipse area $\left(880.06 \pm 980.88 \mathrm{~mm}^{2}\right.$ vs $\left.483.94 \pm 395.55 \mathrm{~mm}^{2}, \mathrm{p}<0.001\right)$, and closed eyes COPY $(9.62 \pm 22.86 \mathrm{~mm}$ vs $6.63 \pm 17.94 \mathrm{~mm}, \mathrm{p}=0.015)$ [TABle 2 \& Figure 2]. Whereas participants in the conventional exercise group had significant improvement in open eyes COPY $(13.00 \pm 16.32 \mathrm{~mm}$ vs $15.17 \pm 16.74 \mathrm{~mm}, \mathrm{p}=0.023)$ and closed eyes COPY $(19.07 \pm 27.68 \mathrm{~mm}$ vs $19.73 \pm 17.02 \mathrm{~mm}, \mathrm{p}=0.047)$ but had significant deterioration in open eyes F-B speed
$(15.63 \pm 9.50 \mathrm{~mm} / \mathrm{s}$ vs $16.13 \pm 11.97 \mathrm{~mm} / \mathrm{s}, \mathrm{p}=0.041)$ and closed eyes F-B speed $(23.41 \pm 17.02 \mathrm{~mm} / \mathrm{s}$ vs $23.53 \pm 11.98 \mathrm{~mm} / \mathrm{s}, \mathrm{p}=0.041$ ) [Table 2 \& Figure 2]. Compared with the conventional exercise group, the Pilates group had significantly greater improvement in MDS-UPDRS $\left(\mathrm{F}_{2.41}=5.347\right.$, $\mathrm{p}=0.026)$, open eyes COP $X\left(\mathrm{~F}_{2.41}=4.678, \mathrm{p}=0.013\right)$, open eyes COPY $\left(\mathrm{F}_{2.41}=4.111, \mathrm{p}=0.021\right)$, open eyes ellipse area $\left(\mathrm{F}_{2.41}=4.228, \mathrm{p}=0.026\right)$, open eyes $\mathrm{F}$-B speed $\left(\mathrm{F}_{2.41}=2.945, \mathrm{p}=0.037\right)$, open eyes $\mathrm{M}$ - $\mathrm{L}$ speed $\left(\mathrm{F}_{2.41}=2.897, \mathrm{p}=0.023\right)$, closed eyes $\mathrm{COPX}\left(\mathrm{F}_{2.41}=3.221\right.$, $\mathrm{p}=0.041)$, closed eyes COPY $\left(\mathrm{F}_{2.41}=4.122, \mathrm{p}=0.003\right)$, and closed eyes F-B speed $\left(\mathrm{F}_{2.41}=3.146, \mathrm{p}=0.041\right)$ [TABLE 2].

From post-intervention to follow-up, participants in the Pilates group had significant deterioration in body mass index $\left(29.25 \pm 2.94 \mathrm{~kg} / \mathrm{m}^{2}\right.$ vs $\left.30.12 \pm 3.31 \mathrm{~kg} / \mathrm{m}^{2}, \quad \mathrm{p}<0.001\right), \quad$ MDS-UPDRS $(43.25 \pm 11.07$ vs $59.13 \pm 17.97, p<0.001)$, open eyes COP X $(-0.75 \pm 3.36 \mathrm{~mm}$ vs $1.43 \pm 5.10 \mathrm{~mm}$, $\mathrm{p}<0.001)$, open eyes COP Y $(3.13 \pm 1.56 \mathrm{~mm}$ vs $10.19 \pm 5.65 \mathrm{~mm}, \mathrm{p}<0.001)$, open eyes $\mathrm{M}-\mathrm{L}$ speed $(10.47 \pm 0.23 \mathrm{~mm} / \mathrm{s}$ vs $10.53 \pm 0.10 \mathrm{~mm} / \mathrm{s}$, $\mathrm{p}=0.047)$, closed eyes COP X $(-3.50 \pm 1.56 \mathrm{~mm}$ vs $-8.81 \pm 4.17 \mathrm{~mm}, \mathrm{p}<0.001)$, closed eyes COP $\mathrm{Y}(6.63 \pm 3.37 \mathrm{~mm}$ vs $7.13 \pm 4.89 \mathrm{~mm}, \mathrm{p}=0.030)$, and closed eyes M-L speed $(15.69 \pm 1.87 \mathrm{~mm} / \mathrm{s}$ vs $17.56 \pm 3.55 \mathrm{~mm} / \mathrm{s}, \quad \mathrm{p}=0.049)$ but maintained the performance in open eyes ellipse area $\left(483.94 \pm 395.55 \mathrm{~mm}^{2}\right.$ vs $950.63 \pm 192.87 \mathrm{~mm}^{2}$, $\mathrm{p}=0.348)$, open eyes F-B speed $(13.59 \pm 8.48 \mathrm{~mm} / \mathrm{s}$ vs $15.75 \pm 20.09 \mathrm{~mm} / \mathrm{s}, \mathrm{p}=0.111)$, closed eyes ellipse area $\left(817.24 \pm 750.00 \mathrm{~mm}^{2}\right.$ vs $1450.00 \pm 329.64 \mathrm{~mm}^{2}$, $\mathrm{p}=0.333)$, and closed eyes F-B speed $(21.69 \pm 17.56 \mathrm{~mm} / \mathrm{s}$ vs $22.78 \pm 15.34 \mathrm{~mm} / \mathrm{s}, \mathrm{p}=0.278)$ [TAble 2 \& Figure 2]. Whereas participants in the conventional exercise group had significant deterioration in body mass index $\left(32.54 \pm 4.65 \mathrm{~kg} / \mathrm{m}^{2}\right.$ vs $\left.32.85 \pm 5.11 \mathrm{~kg} / \mathrm{m}^{2}, \mathrm{p}<0.001\right)$, open eyes COP Y $(15.17 \pm 18.12 \mathrm{~mm}$ vs $16.31 \pm 19.76 \mathrm{~mm}, \mathrm{p}=0.034)$, and closed eyes COP Y $(19.73 \pm 22.54 \mathrm{~mm}$ vs $19.75 \pm 20.43 \mathrm{~mm}, \mathrm{p}=0.009)$ but had significant improvement in open eyes COP X $(-5.80 \pm 3.54 \mathrm{~mm}$ vs $-8.06 \pm 3.50 \mathrm{~mm}, \mathrm{p}=0.036)$ and closed eyes COP $X$ $(-7.10 \pm 6.32 \mathrm{~mm}$ vs $-7.59 \pm 8.67 \mathrm{~mm}, \mathrm{p}=0.040)$ [T ABLE 2 \& Figure 2]. Compared with the conventional exercise group, the Pilates group had significant improvement in MDS-UPDRS $\left(\mathrm{F}_{2.41}=5.597\right.$, $\mathrm{p}=0.015)$, open eyes $\mathrm{COP} X\left(\mathrm{~F}_{2.41}=4.134, \mathrm{p}=0.032\right)$, open eyes $\operatorname{COPY}\left(\mathrm{F}_{2.41}=4.123, \mathrm{p}=0.033\right)$, closed eyes 
TABLE 1

Sociodemographic and clinical characteristics of participants

\begin{tabular}{|c|c|c|c|c|c|}
\hline Sex/age, y & $\begin{array}{l}\text { Body mass } \\
\text { index, } \mathrm{kg} / \mathrm{m}^{2}\end{array}$ & $\begin{array}{c}\text { Hoehn } \\
\text { \&Yahr stage }\end{array}$ & $\begin{array}{l}\text { Time since } \\
\text { diagnosis, y }\end{array}$ & $\begin{array}{l}\text { Education } \\
\text { level }\end{array}$ & Dopamine dose equivalent, mg/day \\
\hline \multicolumn{6}{|c|}{ Pilates group $(n=8)$} \\
\hline $73.25 \pm 7.01$ & $28.55 \pm 2.83$ & $2.38 \pm 0.52$ & $8.00 \pm 6.05$ & & \\
\hline $\mathrm{M} / 80$ & 28.68 & 2 & 11 & Primary & $\begin{array}{l}\text { Levodopa } 600 \text { mg, carbidopa } 125 \text { g, entacapone } 600 \text { mg, } \\
\text { rotigotine } 8 \text { mg, safinamide } 100 \text { mg }\end{array}$ \\
\hline $\mathrm{M} / 75$ & 25.22 & 3 & 2 & University & Levodopa 400 mg, carbidopa 100 mg, rotigotine 4 mg \\
\hline $\mathrm{F} / 80$ & 31.50 & 2 & 4 & None & $\begin{array}{l}\text { Levodopa } 500 \text { mg, carbidopa } 125 \text { mg, entacapone } 400 \text { mg, } \\
\text { rasagiline } 1 \text { mg }\end{array}$ \\
\hline $\mathrm{F} / 63$ & 30.04 & 3 & 12 & Primary & $\begin{array}{l}\text { Levodopa } 900 \mathrm{mg} \text {, carbidopa } 225 \mathrm{~g} \text {, entacapone } 800 \mathrm{mg} \text {, } \\
\text { pramipexole } 3.15 \mathrm{mg} \text {, safinamide } 100 \mathrm{mg}\end{array}$ \\
\hline $\mathrm{M} / 79$ & 25.68 & 2 & 7 & Primary & $\begin{array}{l}\text { Levodopa } 500 \text { mg, carbidopa } 125 \text { mg, entacapone } 400 \text { mg, } \\
\text { rasagiline } 1 \text { mg }\end{array}$ \\
\hline $\mathrm{M} / 69$ & 26.84 & 3 & 20 & Primary & $\begin{array}{l}\text { Levodopa } 900 \text { mg, carbidopa } 225 \mathrm{~g} \text {, entacapone } 800 \mathrm{mg} \text {, } \\
\text { pramipexole } 3.15 \mathrm{mg} \text {, safinamide } 100 \mathrm{mg}\end{array}$ \\
\hline$F / 64$ & 33.15 & 2 & 5 & None & $\begin{array}{l}\text { Levodopa } 900 \text { mg, carbidopa } 225 \mathrm{~g} \text {, entacapone } 800 \mathrm{mg} \text {, } \\
\text { pramipexole } 3.15 \mathrm{mg} \text {, safinamide } 100 \mathrm{mg}\end{array}$ \\
\hline $\mathrm{M} / 76$ & 27.28 & 2 & 3 & Primary & $\begin{array}{l}\text { Levodopa } 900 \text { mg, carbidopa } 225 \mathrm{~g} \text {, entacapone } 800 \mathrm{mg} \text {, } \\
\text { pramipexole } 3.15 \mathrm{mg} \text {, safinamide } 100 \mathrm{mg}\end{array}$ \\
\hline \multicolumn{6}{|c|}{ Conventional exercise group $(n=7)$} \\
\hline $73.71 \pm 7.52$ & $32.54 \pm 4.65$ & $2.43 \pm 0.79$ & $8.29 \pm 4.86$ & & \\
\hline $\mathrm{F} / 69$ & 28.72 & 3 & 16 & Primary & $\begin{array}{l}\text { Levodopa } 900 \text { mg, carbidopa } 225 \mathrm{~g} \text {, entacapone } 800 \mathrm{mg} \text {, } \\
\text { pramipexole } 3.15 \mathrm{mg} \text {, safinamide } 100 \mathrm{mg}\end{array}$ \\
\hline $\mathrm{M} / 74$ & 29.86 & 3 & 6 & Primary & $\begin{array}{l}\text { Levodopa } 900 \text { mg, carbidopa } 225 \mathrm{~g} \text {, entacapone } 800 \text { mg, } \\
\text { pramipexole } 3.15 \text { mg, safinamide } 100 \mathrm{mg}\end{array}$ \\
\hline $\mathrm{M} / 83$ & 26.62 & 2 & 10 & $\begin{array}{l}\text { Vocational } \\
\text { training }\end{array}$ & $\begin{array}{l}\text { Levodopa } 600 \text { mg, carbidopa } 125 \text { g, entacapone } 600 \text { mg, } \\
\text { rotigotine } 8 \text { mg, safinamide } 100 \text { mg }\end{array}$ \\
\hline $\mathrm{F} / 60$ & 34.77 & 3 & 3 & None & $\begin{array}{l}\text { Levodopa } 900 \mathrm{mg} \text {, carbidopa } 225 \mathrm{~g} \text {, entacapone } 800 \mathrm{mg} \text {, } \\
\text { pramipexole } 3.15 \mathrm{mg} \text {, safinamide } 100 \mathrm{mg}\end{array}$ \\
\hline $\mathrm{F} / 80$ & 40.37 & 3 & 13 & None & $\begin{array}{l}\text { Levodopa } 600 \text { mg, carbidopa } 125 \text { g, entacapone } 600 \text { mg, } \\
\text { rotigotine } 8 \text { mg, safinamide } 100 \text { mg }\end{array}$ \\
\hline $\mathrm{F} / 75$ & 32.36 & 2 & 6 & Primary & Levodopa 400 mg, carbidopa 100 mg, rotigotine 4 mg \\
\hline $\mathrm{F} / 75$ & 35.09 & 1 & 4 & None & $\begin{array}{l}\text { Levodopa } 600 \text { mg, carbidopa } 125 \mathrm{~g} \text {, entacapone } 600 \text { mg, } \\
\text { rotigotine } 8 \text { mg, safinamide } 100 \text { mg }\end{array}$ \\
\hline
\end{tabular}

COP X $\left(\mathrm{F}_{2.41}=5.105, \mathrm{p}=0.013\right)$, and closed eyes COPY $\left(\mathrm{F}_{2.41}=6.234, \mathrm{p}=0.001\right)$ [TABLE 2].

\section{DISCUSSION}

Pilates exercise significantly improved clinical symptoms (MDS-UPDRS) of patients with PD, whereas conventional exercise did not. This may be due to the effect of Pilates on motor control. Pilates exercise strengths core muscles (such as transversus abdominis, internal oblique, diaphragm, lumbar multifidus, and pelvic floor muscles) that stabilise the lumbopelvic system, which plays a critical role in the control of trunk movement. ${ }^{20}$ Similarly, Pilates helps to stimulate motor learning and enable better body movement control. ${ }^{24}$ Pilates improves trunk and lower body muscular strength, which are strongly related to physical function, physical impairment, and disability. ${ }^{25}$ Pilates improves the ability to perform activities of daily living in persons with multiple sclerosis. ${ }^{26}$

Pilates exercise significantly improved static balance in our patients with PD. A similar finding was reported in patients with multiple sclerosis. ${ }^{27}$ Body awareness training leads to balance improvements. ${ }^{28}$ People with poor body awareness and maladaptive movement patterns may benefit from Pilates exercises. ${ }^{29}$ Pilates is effective in increasing body awareness in sedentary women by 
TABLE 2

Effects of Pilates and conventional exercise on clinical symptoms and static balance

\begin{tabular}{|c|c|c|c|c|c|c|c|c|c|c|c|c|}
\hline \multirow[t]{4}{*}{ Variable } & \multicolumn{6}{|c|}{ Pre-intervention to post-intervention } & \multicolumn{6}{|c|}{ Post-intervention to follow-up } \\
\hline & \multicolumn{5}{|c|}{ Intragroup analysis } & \multirow{3}{*}{$\begin{array}{l}\text { Intergroup } \\
\text { analysis } \\
\text { Evaluation } \\
\text { x group } \\
\text { (ANOVA) }\end{array}$} & \multicolumn{5}{|c|}{ Intragroup analysis } & \multirow{3}{*}{$\begin{array}{c}\text { Intergroup } \\
\text { analysis } \\
\text { Evaluation } \\
\text { x group } \\
\text { (ANOVA) }\end{array}$} \\
\hline & \multirow{2}{*}{\multicolumn{2}{|c|}{ Mean difference }} & \multicolumn{2}{|c|}{$\begin{array}{c}95 \% \\
\text { confidence } \\
\text { interval }\end{array}$} & \multirow[t]{2}{*}{$\begin{array}{l}\text { Effect } \\
\text { size, d } \\
\text { cohen }\end{array}$} & & \multirow{2}{*}{\multicolumn{2}{|c|}{ Mean difference }} & \multicolumn{2}{|c|}{$\begin{array}{c}95 \% \\
\text { confidence } \\
\text { interval }\end{array}$} & \multirow[t]{2}{*}{$\begin{array}{l}\text { Effect } \\
\text { size, d } \\
\text { cohen }\end{array}$} & \\
\hline & & & Lower & Upper & & & & & Lower & Upper & & \\
\hline \multicolumn{13}{|l|}{ Body mass index, $\mathrm{kg} / \mathrm{m}^{2}$} \\
\hline Pilates & $\begin{array}{c}0.70 \pm 0.96 \\
p=0.076\end{array}$ & $\uparrow$ & -0.09 & 1.16 & $\begin{array}{l}d=0.242 \\
r=-0.120\end{array}$ & $\begin{array}{c}F_{2,41}=0.803 \\
p=0.375\end{array}$ & $\begin{array}{c}0.86 \pm 0.29 \\
p<0.001\end{array}$ & $\downarrow$ & 0.16 & 1.57 & $\begin{array}{l}d=0.278 \\
r=-0.137\end{array}$ & $\begin{array}{c}F_{2,41}=0.442, \\
p=0.510\end{array}$ \\
\hline Conventional exercise & $\begin{array}{c}0.31 \pm 1.29 \\
p=0.185\end{array}$ & $\downarrow$ & -1.04 & 1.66 & $\begin{array}{l}d=0.064 \\
r=-0.032\end{array}$ & & $\begin{array}{c}0.79 \pm 0.27 \\
p<0.001\end{array}$ & $\downarrow$ & -0.07 & 1.65 & $\begin{array}{c}d=0.272 \\
r=0.135\end{array}$ & \\
\hline \multicolumn{13}{|l|}{$\begin{array}{l}\text { Movement Disorder Society } \\
\text { Unified Parkinson's Disease } \\
\text { Rating Scale }\end{array}$} \\
\hline Pilates & $\begin{array}{c}15.13 \pm 10.87 \\
p<0.001\end{array}$ & $\uparrow$ & -24.21 & -6.04 & $\begin{array}{l}\mathrm{d}=0.912 \\
\mathrm{r}=0.451\end{array}$ & $\begin{array}{c}F_{2.41}=5.347 \\
p=0.026\end{array}$ & $\begin{array}{c}15.87 \pm 3.27 \\
p<0.001\end{array}$ & $\downarrow$ & 8.14 & 23.61 & $\begin{array}{l}d=0.964 \\
r=-0.469\end{array}$ & $\begin{array}{c}\mathrm{F}_{2,41}=5.597 \\
\mathrm{p}=0.015\end{array}$ \\
\hline Conventional exercise & $\begin{array}{c}3.83 \pm 7.03 \\
p=0.332\end{array}$ & $\downarrow$ & -3.54 & 11.21 & $\begin{array}{l}d=0.211 \\
r=-0.105\end{array}$ & & $\begin{array}{c}2.25 \pm 2.92 \\
p=0.101\end{array}$ & $\downarrow$ & -7.06 & 11.56 & $\begin{array}{c}d=0.172 \\
r=0.086\end{array}$ & \\
\hline \multicolumn{13}{|l|}{ Open eyes COP X, mm } \\
\hline Pilates & $\begin{array}{c}0.94 \pm 15.84 \\
p=0.032\end{array}$ & $\uparrow$ & -14.18 & 12.30 & $\begin{array}{l}d=0.211 \\
r=-0.105\end{array}$ & $\begin{array}{c}F_{2,41}=4.678 \\
p=0.013\end{array}$ & $\begin{array}{c}0.68 \pm 4.23 \\
p<0.001\end{array}$ & $\downarrow$ & -9.33 & 10.70 & $\begin{array}{l}d=0.340 \\
r=0.167\end{array}$ & $\begin{array}{c}\mathrm{F}_{2,41}=4.134, \\
\mathrm{p}=0.032\end{array}$ \\
\hline Conventional exercise & $\begin{array}{c}3.58 \pm 10.45 \\
p=0.076\end{array}$ & $\downarrow$ & -14.55 & 7.38 & $\begin{array}{l}d=0.001 \\
r=0.001\end{array}$ & & $\begin{array}{c}2.25 \pm 3.52 \\
p=0.036\end{array}$ & $\uparrow$ & -13.47 & 8.97 & $\begin{array}{l}d=0.011 \\
r=0.005\end{array}$ & \\
\hline \multicolumn{13}{|l|}{ Open eyes COP Y, mm } \\
\hline Pilates & $\begin{array}{c}4.00 \pm 17.08 \\
p<0.001\end{array}$ & $\uparrow$ & -10.28 & 18.28 & $\begin{array}{c}d=0.428 \\
r=0.209\end{array}$ & $\begin{array}{c}\mathrm{F}_{2,41}=4.111 \\
\mathrm{p}=0.021\end{array}$ & $\begin{array}{c}7.06 \pm 0.83 \\
p<0.001\end{array}$ & $\downarrow$ & -13.77 & -0.35 & $\begin{array}{c}d=0.488 \\
r=0.237\end{array}$ & $\begin{array}{c}\mathrm{F}_{2,41}=4.123 \\
\mathrm{p}=0.033\end{array}$ \\
\hline Conventional exercise & $\begin{array}{c}2.00 \pm 33.39 \\
p=0.023\end{array}$ & $\uparrow$ & -33.04 & 37.04 & $\begin{array}{l}d=0.131 \\
r=-0.065\end{array}$ & & $\begin{array}{c}0.37 \pm 8.21 \\
p=0.034\end{array}$ & $\downarrow$ & -26.53 & 25.78 & $\begin{array}{l}d=0.060 \\
r=-0.030\end{array}$ & \\
\hline \multicolumn{13}{|c|}{ Open eyes ellipse area, $\mathrm{mm}^{2}$} \\
\hline Pilates & $\begin{array}{c}13.06 \pm 27.96 \\
p<0.001\end{array}$ & $\uparrow$ & -36.43 & 10.31 & $\begin{array}{c}d=0.529 \\
r=0.256\end{array}$ & $\begin{array}{c}F_{2,41}=4.228 \\
p=0.026\end{array}$ & $\begin{array}{c}0.68 \pm 2.12 \\
p=0.348\end{array}$ & $\uparrow$ & -4.33 & 5.71 & $\begin{array}{l}d=0.899 \\
r=-0.599\end{array}$ & $\begin{array}{c}\mathrm{F}_{2,41}=1.030 \\
\mathrm{p}=0.316\end{array}$ \\
\hline Conventional exercise & $\begin{array}{c}5.33 \pm 5.49 \\
p=0.321\end{array}$ & $\downarrow$ & -11.10 & 0.43 & $\begin{array}{l}d=0.003 \\
r=-0.001\end{array}$ & & $\begin{array}{c}3.50 \pm 1.63 \\
p=0.289\end{array}$ & $\uparrow$ & -1.70 & 8.70 & $\begin{array}{l}d=0.112 \\
r=-0.056\end{array}$ & \\
\hline \multicolumn{13}{|c|}{ Open eyes F-B speed, mm/s } \\
\hline Pilates & $\begin{array}{l}0.50 \pm 0.65 \\
p=0.056\end{array}$ & $\uparrow$ & -1.05 & 0.05 & $\begin{array}{c}d=0.169 \\
r=0.084\end{array}$ & $\begin{array}{c}\mathrm{F}_{2,44}=2.945 \\
\mathrm{p}=0.037\end{array}$ & $\begin{array}{c}0.06 \pm 0.14 \\
p=0.111\end{array}$ & $\uparrow$ & -0.41 & 0.29 & $\begin{array}{l}d=0.140 \\
r=-0.069\end{array}$ & $\begin{array}{c}\mathrm{F}_{2,41}=1.134 \\
\mathrm{p}=0.342\end{array}$ \\
\hline Conventional exercise & $\begin{array}{c}0.75 \pm 0.27 \\
p=0.041\end{array}$ & $\downarrow$ & -1.04 & -0.46 & $\begin{array}{l}d=0.046 \\
r=-0.023\end{array}$ & & $\begin{array}{c}0.37 \pm 0.12 \\
p=0.267\end{array}$ & $\downarrow$ & -0.02 & 0.77 & $\begin{array}{l}d=0.019 \\
r=-0.009\end{array}$ & \\
\hline \multicolumn{13}{|c|}{ Open eyes M-L speed, mm/s } \\
\hline Pilates & $\begin{array}{c}0.50 \pm 0.65 \\
p=0.210\end{array}$ & $\uparrow$ & -1.05 & 0.05 & $\begin{array}{c}d=0.343 \\
r=0.169\end{array}$ & $\begin{array}{c}F_{2,41}=2.897 \\
p=0.023\end{array}$ & $\begin{array}{c}0.06 \pm 0.11 \\
p=0.047\end{array}$ & $\downarrow$ & -0.21 & 0.33 & $\begin{array}{l}d=0.587 \\
r=-0.281\end{array}$ & $\begin{array}{c}\mathrm{F}_{2,41}=1.002, \\
\mathrm{p}=0.431\end{array}$ \\
\hline Conventional exercise & $\begin{array}{c}0.75 \pm 0.52 \\
p=0.178\end{array}$ & $\downarrow$ & -1.30 & -0.20 & $\begin{array}{l}d=0.211 \\
r=-0.105\end{array}$ & & $\begin{array}{c}0.37 \pm 0.23 \\
p=0.187\end{array}$ & $\downarrow$ & -0.39 & 1.14 & $\begin{array}{l}d=0.243 \\
r=-0.120\end{array}$ & \\
\hline \multicolumn{13}{|l|}{ Closed eyes COP X, mm } \\
\hline Pilates & $\begin{array}{c}0.31 \pm 16.92 \\
p=0.099\end{array}$ & $\uparrow$ & -14.46 & 13.83 & $\begin{array}{l}d=0.175 \\
r=-0.087\end{array}$ & $\begin{array}{c}F_{2,41}=3.221 \\
p=0.041\end{array}$ & $\begin{array}{c}5.31 \pm 4.57 \\
p<0.001\end{array}$ & $\downarrow$ & -5.51 & 16.14 & $\begin{array}{l}d=0.434 \\
r=-0.212\end{array}$ & $\begin{array}{c}\mathrm{F}_{2,41}=5.105 \\
\mathrm{p}=0.013\end{array}$ \\
\hline Conventional exercise & $\begin{array}{c}5.00 \pm 7.94 \\
p=0.201\end{array}$ & $\downarrow$ & -13.34 & 3.34 & $\begin{array}{l}d=0.006 \\
r=-0.003\end{array}$ & & $\begin{array}{c}1.37 \pm 3.35 \\
p=0.040\end{array}$ & $\uparrow$ & -12.04 & 9.29 & $\begin{array}{c}d=0.020 \\
r=0.010\end{array}$ & \\
\hline \multicolumn{13}{|l|}{ Closed eyes COP Y, mm } \\
\hline Pilates & $\begin{array}{c}7.88 \pm 19.45 \\
p=0.015\end{array}$ & $\uparrow$ & -8.39 & 24.14 & $\begin{array}{l}d=0.145 \\
r=-0.072\end{array}$ & $\begin{array}{c}F_{2,41}=4.112 \\
p=0.003\end{array}$ & $\begin{array}{c}0.50 \pm 6.59 \\
p=0.030\end{array}$ & $\downarrow$ & -16.09 & 15.09 & $\begin{array}{l}d=0.284 \\
r=0.140\end{array}$ & $\begin{array}{c}\mathrm{F}_{2,41}=6.234 \\
\mathrm{p}=0.001\end{array}$ \\
\hline Conventional exercise & $\begin{array}{c}12.42 \pm 7.96 \\
p=0.047\end{array}$ & $\uparrow$ & 4.06 & 20.77 & $\begin{array}{l}d=0.028 \\
r=-0.014\end{array}$ & & $\begin{array}{c}-25.75 \pm 17.05 \\
p=0.009\end{array}$ & $\downarrow$ & -80.01 & 28.51 & $\begin{array}{c}d=0.009 \\
r=0.004\end{array}$ & \\
\hline \multicolumn{13}{|c|}{ Closed eyes ellipse area, $\mathrm{mm}^{2}$} \\
\hline Pilates & $\begin{array}{c}13.06 \pm 27.96 \\
p=0.064\end{array}$ & $\uparrow$ & -36.43 & 10.31 & $\begin{array}{c}d=0.274 \\
r=0.136\end{array}$ & $\begin{array}{c}F_{2,44}=1.129 \\
p=0.294\end{array}$ & $\begin{array}{c}0.68 \pm 2.12 \\
p=0.333\end{array}$ & $\uparrow$ & -4.33 & 5.71 & $\begin{array}{l}d=0.892 \\
r=-0.479\end{array}$ & $\begin{array}{c}\mathrm{F}_{2,41}=0.078 \\
\mathrm{p}=0.781\end{array}$ \\
\hline Conventional exercise & $\begin{array}{c}5.33 \pm 5.49 \\
p=0.342\end{array}$ & $\downarrow$ & -11.10 & 0.43 & $\begin{array}{c}d=0.028 \\
r=0.014\end{array}$ & & $\begin{array}{c}3.50 \pm 1.63 \\
p=0.301\end{array}$ & $\downarrow$ & -1.70 & 8.70 & $\begin{array}{l}d=0.025 \\
r=-0.012\end{array}$ & \\
\hline Closed eyes F-B speed, mr & & & & & & & & & & & & \\
\hline Pilates & $\begin{array}{c}11.06 \pm 32.34 \\
p=0.058\end{array}$ & $\uparrow$ & -15.97 & 38.10 & $\begin{array}{l}d=0.046 \\
r=-0.023\end{array}$ & $\begin{array}{c}F_{2,41}=3.146 \\
p=0.041\end{array}$ & $\begin{array}{c}0.31 \pm 4.03 \\
p=0.278\end{array}$ & $\uparrow$ & -9.23 & 9.86 & $\begin{array}{l}d=0.066 \\
r=-0.033\end{array}$ & $\begin{array}{c}\mathrm{F}_{2,41}=1.456 \\
\mathrm{p}=0.221\end{array}$ \\
\hline Conventional exercise & $\begin{array}{c}10.92 \pm 13.57 \\
p=0.041\end{array}$ & $\downarrow$ & -3.32 & 25.16 & $\begin{array}{l}d=0.008 \\
r=-0.004\end{array}$ & & $\begin{array}{c}0.75 \pm 4.90 \\
p=0.067\end{array}$ & $\downarrow$ & -16.36 & 14.86 & $\begin{array}{c}d=0.026 \\
r=-0.013\end{array}$ & \\
\hline Closed eyes M-L speed, mi & & & & & & & & & & & & \\
\hline Pilates & $\begin{array}{c}3.38 \pm 16.92 \\
p=0.051\end{array}$ & $\uparrow$ & -10.77 & 17.52 & $\begin{array}{l}d=0.162 \\
r=-0.080\end{array}$ & $\begin{array}{c}F_{2,41}=1.756 \\
p=0.132\end{array}$ & $\begin{array}{c}-1.43 \pm 2.80 \\
p=0.049\end{array}$ & $\downarrow$ & -8.06 & 5.19 & $\begin{array}{l}d=0.211 \\
r=0.105\end{array}$ & $\begin{array}{c}\mathrm{F}_{2,41}=2.001 \\
\mathrm{p}=0.065\end{array}$ \\
\hline Conventional exercise & $\begin{array}{c}7.92 \pm 10.66 \\
p=0.221\end{array}$ & $\uparrow$ & -2.91 & 18.55 & $\begin{array}{l}d=0.136 \\
r=-0.068\end{array}$ & & $\begin{array}{c}-0.50 \pm 5.27 \\
p=0.198\end{array}$ & $\downarrow$ & -17.29 & 16.29 & $\begin{array}{c}d=0.057 \\
r=0.028\end{array}$ & \\
\hline
\end{tabular}

Abbreviation: $\downarrow$, deterioration; $\uparrow$, improvement; COP X, centre of pressure of the body in the frontal plane; COP Y, centre of pressure of the body in the sagittal plane; F-B speed, speed of oscillation movement of the centre of pressure in the sagittal plane (forward-backward); and M-L speed, speed of oscillation movement of the centre of pressure in the frontal plane (medium-lateral) 


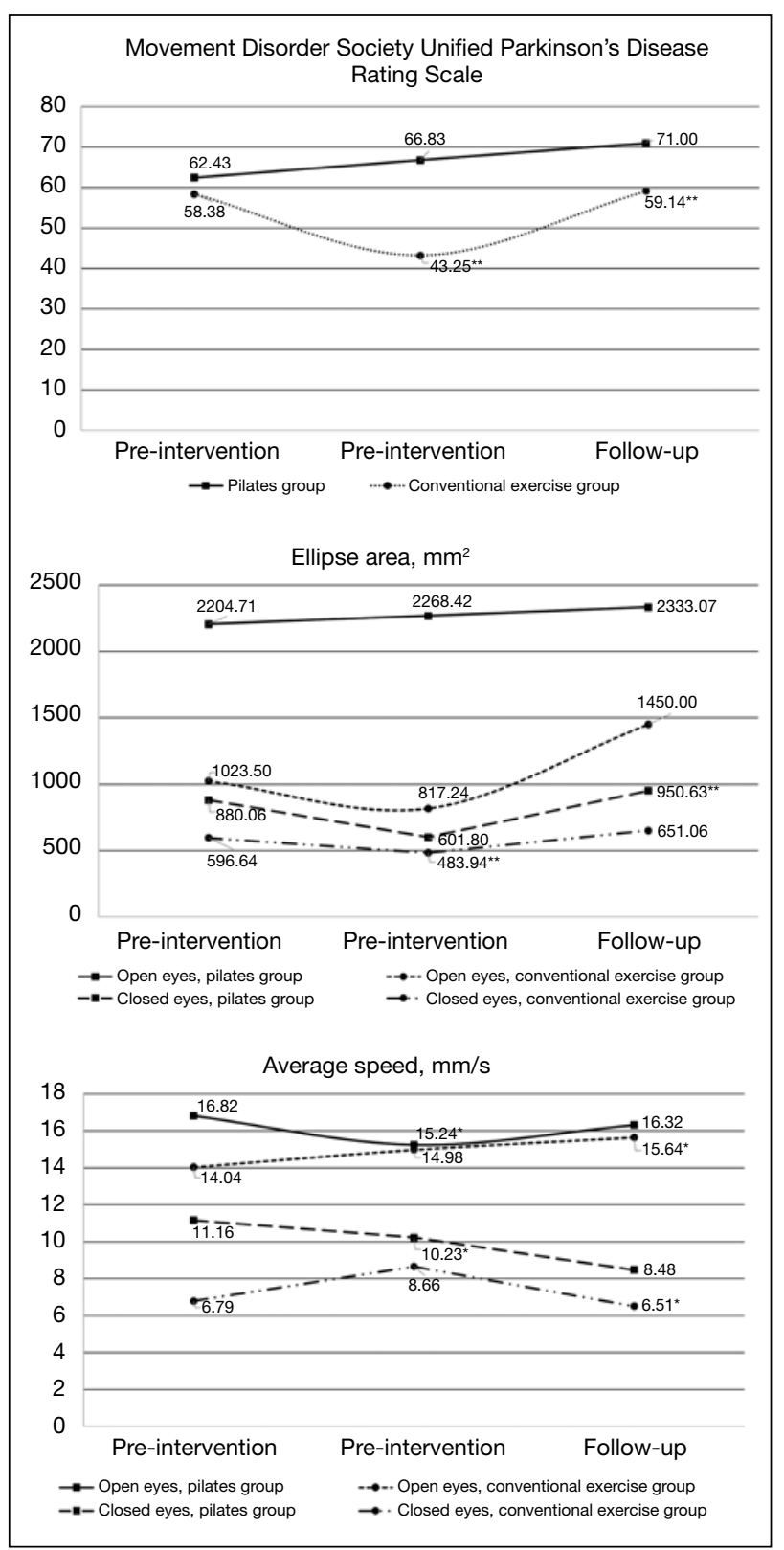

FiguRE 2. Comparison of static balance parameters of the Pilates and conventional exercise groups from preintervention to follow-up

stimulating interoceptive channels that contribute to the perception of the body and movements. ${ }^{30}$ Proprioception plays a key role in postural stability, as adequate balance relies on the accurate perception of physical stimuli provided by the somatosensory and vestibular systems. ${ }^{31}$ Therefore, the positive effects of Pilates on static balance could be due to enhanced sensorimotor mapping of body movements (locomotor and vestibular). Specific muscles responsible for balance were stimulated by Pilates exercise. A Pilates programme focusing on core body exercises and breathing control facilitates the activation of deep trunk muscles (ie, transversus abdominis, diaphragm, and multifidus) and pelvic floor muscles and thus improves postural stability. ${ }^{32}$ Increased muscular fitness leads to core stability and strength. ${ }^{33}$ Nonetheless, in the present study, not all stabilometer parameters improved significantly after Pilates exercise. This may be because the Pilates exercise was performed in a seated or supine position, and limited balance stimulation was invoked during exercise in the standing position. ${ }^{34}$

In a study of 10 patients with PD, 6 weeks of Pilates exercise significantly improved motor impairment but not static balance. ${ }^{17}$ This may be due to the short duration of the programme and the difference type of Pilates exercises. In the present study, the beneficial effects of Pilates disappeared shortly after the intervention ended. This may be because participants were told not to exercise during the follow-up period. Indeed, their body mass index significantly increased at follow-up, suggesting a lack of physical activity. In contrast, in a study of community-dwelling older people, significant improvements in static balance were maintained 5 weeks after the intervention ended. ${ }^{35}$ Similarly, in patients with $\mathrm{PD}$ taking part in exercise interventions, motor and balance improvements did not usually return to baseline levels at follow-up..$^{35,36}$

Participants in the conventional exercise group did not have significant improvement in clinical symptoms or static balance after the intervention, although conventional exercise had been reported to have positive effects on motor impairments and balance $^{37}$ and to be a useful rehabilitation strategy in patients with PD. ${ }^{38}$ The lack of improvement in the conventional exercise group could be due to a ceiling effect on participants, as most participants had regularly taken physical activity programmes organised by the association and thus conventional exercise may not provide new stimulus to participants.

There were limitations to the study. The sample size was small and the participants were not representative of patients with PD in terms of participation in exercise and active lifestyle. Analysis of variance was used, but multivariate analysis of variance may be more appropriate, because patients with different stages of PD were included and the main variable was static balance. 
These methodological weak points might limit generalisation of the findings.

\section{CONCLUSION}

Pilates exercise is a useful rehabilitation strategy for people with mild to moderate PD, with positive effect on motor impairment and static balance. Further study with a more representative sample is needed to confirm these results.

\section{CONTRIBUTORS}

All authors designed the study, acquired the data, analysed the data, drafted the manuscript, and critically revised the manuscript for important intellectual content. All authors had full access to the data, contributed to the study, approved the final version for publication, and take responsibility for its accuracy and integrity.

\section{CONFLICTS OF INTEREST}

All authors have disclosed no conflicts of interest.

\section{FUNDING/SUPPORT}

This study received no specific grant from any funding agency in the public, commercial, or notfor-profit sectors.

\section{DATA AVAILABILITY}

All data generated or analysed during the present study are available from the corresponding author on reasonable request.

\section{ETHICS APPROVAL}

This study was approved by the Ethics Committee of the University of Vigo, Pontevedra, Spain (reference: EC2015/484). The patients were treated in accordance with the tenets of the Declaration of Helsinki. The patients provided written informed consent for all treatments and procedures.

\section{ACKNOWLEDGEMENT}

We would like to thank the Parkinson's Association of Pontevedra, Spain for its participation.

\section{REFERENCES}

1. Alexander GE. Biology of Parkinson's disease: pathogenesis and pathophysiology of a multisystem neurodegenerative disorder. Dialogues Clin Neurosci 2004;6:259-80. Crossref

2. Jankovic J. Parkinson's disease: clinical features and diagnosis. J Neurol Neurosurg Psychiatry 2008;79:368-76. Crossref

3. Di Giulio I, St George RJ, Kalliolia E, Peters AL, Limousin P, Day BL. Maintaining balance against force perturbations: impaired mechanisms unresponsive to levodopa in Parkinson's disease. J Neurophysiol 2016;116:493-502. Crossref

4. Dibble LE, Addison O, Papa E. The effects of exercise on balance in persons with Parkinson's disease: a systematic review across the disability spectrum. J Neurol Phys Ther 2009;33:14-26. Crossref

5. Mak MK, Wong-Yu IS, Shen X, Chung CL. Long-term effects of exercise and physical therapy in people with Parkinson disease. Nat Rev Neurol 2017;13:689-703. Crossref

6. Ahlskog JE. Aerobic exercise: evidence for a direct brain effect to slow Parkinson disease progression. Mayo Clin Proc 2018;93:36072. Crossref

7. Goodwin VA, Richards SH, Taylor RS, Taylor AH, Campbell JL. The effectiveness of exercise interventions for people with Parkinson's disease: a systematic review and meta-analysis. Mov Disord 2008;23:631-40. Crossref

8. Abbruzzese G, Marchese R, Avanzino L, Pelosin E. Rehabilitation for Parkinson's disease: current outlook and future challenges. Parkinsonism Relat Disord 2016;22 Suppl 1:S60-4. Crossref

9. Ayan Pérez C, Cancela JM. Effectiveness of water-based exercise in people living with Parkinson's disease: a systematic review. Eur Rev Aging Phys Act 2014;11:107-18. Crossref

10. Klamroth S, Steib S, Devan S, Pfeifer K. Effects of exercise therapy on postural instability in Parkinson disease: a meta-analysis. J Neurol Phys Ther 2016;40:3-14. Crossref

11. Kalron A, Rosenblum U, Frid L, Achiron A. Pilates exercise training vs. physical therapy for improving walking and balance in people with multiple sclerosis: a randomized controlled trial. Clin Rehabil 2017;31:319-28. Crossref

12. Kamioka H, Tsutani K, KatsumataY, et al. Effectiveness of Pilates exercise: a quality evaluation and summary of systematic reviews based on randomized controlled trials. Complement Ther Med 2016;25:1-19. Crossref

13. King LA, Horak FB. Delaying mobility disability in people with Parkinson disease using a sensorimotor agility exercise program. Phys Ther 2009;89:384-93. Crossref

14. Newell D, Shead V, Sloane L. Changes in gait and balance parameters in elderly subjects attending an 8-week supervised Pilates programme. J Bodyw Mov Ther 2012;16:549-54. Crossref

15. Hyun J, Hwangbo K, Lee CW. The effects of pilates mat exercise on the balance ability of elderly females. J Phys Ther Sci 2014;26:291-3. Crossref

16. Robinson K, Dennison A, Roalf D, et al. Falling risk factors in Parkinson's disease. NeuroRehabilitation 2005;20:169-82. Crossref

17. Johnson LG, Putrino D, James I, et al. The effects of a supervised Pilates training program on balance in Parkinson's disease. Adv Parkinsons Dis 2013;2:58-61. Crossref

18. King LA, Salarian A, Mancini M, et al. Exploring outcome measures for exercise intervention in people with Parkinson's disease. Parkinsons Dis 2013;2013:572134. Crossref

19. Santamato A, Ranieri M, Panza F, et al. Botulinum toxin type A and a rehabilitation program in the treatment of Pisa syndrome in Parkinson's disease. J Neurol 2010;257:139-41. Crossref

20. Cancela JM, Mollinedo Cardalda I, Ayán C, de Oliveira IM. Feasibility and efficacy of mat Pilates on people with mild-tomoderate Parkinson's disease: a preliminary study. Rejuvenation Res 2018;21:109-16. Crossref

21. Mollinedo-Cardalda I, Cancela-Carral JM, Vila-Suárez MH. 
Effect of a mat Pilates program with TheraBand on dynamic balance in patients with Parkinson's disease: feasibility study and randomized controlled trial. Rejuvenation Res 2018;21:42330. Crossref

22. Martínez-Martín P, Rodríguez-Blázquez C, Álvarez-Sánchez M, et al. Expanded and independent validation of the Movement Disorder Society-Unified Parkinson's Disease Rating Scale (MDS-UPDRS). J Neurol 2013;260:228-36. Crossref

23. Ayán C, Cancela JM, Gutiérrez-Santiago A, Prieto I. Effects of two different exercise programs on gait parameters in individuals with Parkinson's disease: a pilot study. Gait Posture 2014;39:64851. Crossref

24. Phrompaet S, Paungmali A, Pirunsan U, Sitilertpisan, P. Effects of pilates training on lumbo-pelvic stability and flexibility. Asian J Sports Med 2011;2:16-22. Crossref

25. Lange C, Unnithan VB, Larkam E, Latta PM. Maximizing the benefits of Pilates-inspired exercise for learning functional motor skills. J Bodyw Mov Ther 2000;4:99-108. Crossref

26. Markovic G, Sarabon N, Greblo Z, KrizanicV. Effects of feedbackbased balance and core resistance training vs. Pilates training on balance and muscle function in older women: a randomizedcontrolled trial. Arch Gerontol Geriatr 2015;61:117-23. Crossref

27. Küçük F, Kara B, Poyraz EÇ, İdiman E. Improvements in cognition, quality of life, and physical performance with clinical Pilates in multiple sclerosis: a randomized controlled trial. J Phys Ther Sci 2016;28:761-8. Crossref

28. de Oliveira Francisco C, de Almeida Fagundes A, Gorges B. Effects of Pilates method in elderly people: systematic review of randomized controlled trials. J Bodyw Mov Ther 2015;19:5008. Crossref

29. Bang DH, Cho HS. Effect of body awareness training on balance and walking ability in chronic stroke patients: a randomized controlled trial. J Phys Ther Sci 2016;28:198-201. Crossref

30. Wells C, Kolt GS, Marshall P, Bialocerkowski A. Indications, benefits, and risks of Pilates exercise for people with chronic low back pain: a Delphi survey of Pilates-trained physical therapists. Phys Ther 2014;94:806-17. Crossref

31. Tolnai N, Szabó Z, Köteles F, Szabo A. Physical and psychological benefits of once-a-week Pilates exercises in young sedentary women: a 10-week longitudinal study. Physiol Behav 2016;163:211-8. Crossref

32. La Touche R, Escalante K, Linares MT. Treating non-specific chronic low back pain through the Pilates Method. J Bodyw Mov Ther 2008;12:364-70. Crossref

33. Donath L, Roth R, Hürlimann C, Zahner L, Faude O. Pilates vs. balance training in health community-dwelling seniors: a 3-arm, randomized controlled trial. Int J Sports Med 2016;37:20210. Crossref

34. Guclu-Gunduz A, Citaker S, Irkec C, Nazliel B, Batur-Caglavan HZ. The effects of pilates on balance, mobility and strength in patients with multiple sclerosis. NeuroRehabilitation 2014;34:337-42. Crossref

35. Bird ML, Hill KD, Fell JW. A randomized controlled study investigating static and dynamic balance in older adults after training with Pilates. Arch Phys Med Rehabil 2012;93:43-9. Crossref

36. Schmitz-Hübsch T, Pyfer D, Kielwein K, Fimmers R, Klockgether T, Wüllner U. Qigong exercise for the symptoms of Parkinson's disease: a randomized, controlled pilot study. Mov Disord 2006;21:543-8. Crossref

37. Wong-Yu IS, Mak MK. Multi-dimensional balance training programme improves balance and gait performance in people with Parkinson's disease: a pragmatic randomized controlled trial with 12-month follow-up. Parkinsonism Relat Disord 2015;21:615-21. Crossref

38. Volpe D, Giantin MG, Maestri R, Frazzitta G. Comparing the effects of hydrotherapy and land-based therapy on balance in patients with Parkinson's disease: a randomized controlled pilot study. Clin Rehabil 2014;28:1210-7. Crossref 